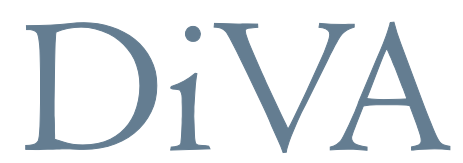

http://uu.diva-portal.org

This is an author produced version of a paper published in Journal of clinical psychology in medical settings. This paper has been peerreviewed but does not include the final publisher proof-corrections or journal pagination.

Citation for the published paper:

Annika Lindahl Norberg

"Burnout in Mothers and Fathers of Children Surviving Brain Tumour" Journal of clinical psychology in medical settings, 2007, Vol. 14, Issue 2, pp. $130-137$

URL: http://dx.doi.org/10.1007/s10880-007-9063-x

The original publication is available at www.springerlink.com. Access to the original publication may require subscription. 


\title{
Burnout in Mothers and Fathers of Children Surviving Brain Tumour.
}

\author{
Annika Lindahl Norberg ${ }^{1}$ \\ Running head: Burnout in parents of brain tumour survivors \\ ${ }^{1}$ Department of Woman and Child Health, Childhood Cancer Research Unit, \\ Karolinska Institutet, Stockholm, Sweden. Correspondence should be addressed to Annika Lindahl \\ Norberg, Ph.D., Childhood Cancer Research Unit, Astrid Lindgren Children's Hospital Q6:05, \\ SE-171 76 Stockholm, Sweden, e-mail: annika.lindahl@ki.se
}

\begin{abstract}
The aim of this paper was to investigate the occurrence of burnout among parents of brain tumour survivors. Burnout was assessed in 24 mothers and 20 fathers of childhood brain tumour survivors, using the Shirom-Melamed Burnout Questionnaire. Parents of children with no history of chronic or serious diseases served as a reference group.

Mothers' burnout scores were significantly higher compared with reference mothers. For fathers, no relation between burnout and being a parent of a brain tumour survivor was established, despite a nonsignificant tendency in the same direction as for the mothers.

In conclusion, the parenting burden after a child's brain tumour may include chronic strain, and this may be particularly taxing for mothers. The findings encourage further studies of psychological exhaustion among parents of children with special needs.
\end{abstract}

KEY WORDS: Parents, childhood cancer survivors, burnout, stress

Introduction

When a child is diagnosed as having cancer, most parents experience a crisis. Previous studies have demonstrated a variety of parental reactions, and occasionally even higher levels of distress than in the patients themselves (e.g. Kazak et al., 2004). Moreover, cancer treatment of a child has been assumed to be a period of chronic stress for the parents (Barrera et al., 2004; Hoekstra-Weebers, Jaspers, Kamps, \& Klip, 2001; Miller, Cohen, \& Ritchey, 2002). Generic distress, such as anxiety, depression, and general psychological symptoms, is often elevated during the active phase of the child's treatment, but subsequently declines and is within a few years comparable to the levels in a community sample (Frank, Brown, Blount, \& Bunke, 2001; Grootenhuis \& Last, 1997; Sawyer, Antoniou, Toogood, Rice, \& Baghurst, 2000; Wijnberg-Williams, Kamps, Klip, \& Hoekstra-Weebers, 2006). Only in a minority of parents does the child's illness appear linked to enduring psychopathology. 
On the other hand, subclinical reactions related to the child's cancer, for example symptoms of disease-related traumatic stress, helplessness, uncertainty, and low control, are reported to occur among parents even many years after completed treatment (Barakat et al., 1997; Grootenhuis \& Last, 1997; Kazak et al., 2004; Kazak et al., 1997; Leventhal-Belfer, Bakker, \& Russo, 1993; Lindahl Norberg, Lindblad, \& Boman, 2005; Van Dongen-Melman et al., 1995). It has been suggested that enduring parental reactions may indicate the processing of a past trauma, experienced during active treatment (e.g. Brown, Madan-Swain, \& Lambert, 2003; Kazak et al., 2004).

However, such reactions may also reflect the influence of present stressors (Lindahl Norberg et al., 2005; Phipps, Long, Hudson, \& Rai, 2005) although current parental stressors after a child's cancer have rarely been systematically surveyed. Initial feelings of euphoria when the child has been discharged from treatment soon fade, as an awareness of the threat of relapse becomes marked (Quin, 2004; Van Dongen-Melman, Van Zuuren, \& Verhulst, 1998). This stressor, known by Koocher's and O'Malley's (1981) now classical expression The Damocles Syndrome, is usually the only ongoing cancer-related stressor cited in studies involving parents after a child's cancer treatment. Nevertheless, Forinder (2004), studying parents' everyday lives after a bone marrow transplant, notes that the child's present somatic and psychological state has vital impact on the parents' everyday life. Similarly, impairments due to other aggressive cancer therapies, as well as to the illness itself, can be assumed to influence parental stress.

Treatment for childhood brain tumour has become more effective but also more aggressive, and remaining sequelae are relatively common, particularly after irradiation therapy, but also after surgery and chemotherapy (Anderson, 2003; Carpentieri, Meyer et al., 2003; Carpentieri, Waber et al., 2003). In addition, the tumour itself may have adverse consequences. A number of studies have described impaired verbal memory, visuospatial organization and motor function in a significant subgroup of children after treatment for brain tumours (Anderson, 2003; Duffner, 2004; Mulhern \& Butler, 2004). Neurocognitive deficits occurring after completion of treatment are generally assumed to be chronic (Mulhern \& Butler, 2004) but ongoing cognitive remediation training is essential, as it may improve the conditions (van't Hooft et al., 2005). In addition to neurocognitive sequelae, endocrine dysfunction is also frequent, leading to growth failure and disturbed sexual development (Anderson, 2003; Duffner, 2004). Survivors of pediatric brain tumours also seem to risk difficulties with social relationships (Fuemmeler, Mullins, \& Marx, 2001; Mabbott et al., 2005; Mulhern et al., 2005). In the long run, there is a risk that brain tumour survivors will face limitations in their lives regarding educational achievement, marriage, parenthood and employment (Anderson, 2003; Mulhern, Merchant, Gajjar, Reddick, \& Kun, 2004). The impairment of the prerequisites for a good quality of life in brain tumour survivors is reflected by demographical data, as well as by reports from parents (Carpentieri, Meyer et al., 2003; Fuemmeler et al., 2001). Remarkably enough, despite the more or less obvious deficits, the survivors themselves frequently report a quality of life on par with their healthy peers (Carpentieri, Meyer et al., 2003; Fuemmeler et al., 2001). It has been suggested that this discrepancy can be attributed to the parents' wider awareness of the sequelae and restrictions of the child's life (Radcliffe, Bennett, Kazak, Foley, \& Phillips, 1996).

In summary, parents of children surviving brain tumours are likely to experience long-term strain related to the child's disease. Since the negative consequences of chronic stress can be serious (Strike \& Steptoe, 2004; Toker, Shirom, Shapira, Berliner, \& Melamed, 2005), further examination of chronic stress among parents is justified.

One of the most widely studied aspects of stress-related psychological exhaustion is burnout. The burnout syndrome, a constellation of symptoms including physical, emotional and cognitive exhaustion, is a consequence of longstanding stress exposure (Melamed, Kushnir, \& Shirom, 1992; Shirom, Westman, Shamai, \& Carel, 1997). Originally regarded as exclusively an effect of work stress (Maslach, Schaufeli, \& Leiter, 2001), burnout may follow long-term stress irrespective of its source (Swedish National Board of Health and Welfare, 2003). For example, it has been seen among parents with an excessive parenting burden due to a child's chronic illness (Weiss, 2002).

The general aim of the present study was to examine whether the concept of burnout was applicable in the context of parenting a child who has survived a brain tumour. This paper describes a straightforward investigation of the occurrence of burnout among mothers and fathers of brain tumour survivors, including a comparison of burnout between these parents and parents of children with no 
history of chronic or serious diseases. Self-report questionnaires were used to assess burnout in a cross-sectional sample of parents whose children had completed treatment for brain tumour. Burnout was assessed on one single occasion in each individual parent.

\section{Method}

\section{Participants}

This report includes data from 44 parents ( 24 mothers and 20 fathers), a subset of the data collected during a larger study investigating stress among parents of children who had completed treatment for CNS tumours. The larger study had a longitudinal design, including two assessments (T1 and T2) at an interval of approximately seven months. Burnout was assessed only on the second occasion (T2), and consequently only data from $\mathrm{T} 2$ are considered in the present paper.

Inclusion criteria for invitation were: parents of children diagnosed with malignant brain tumour; child should be disease-free and the cancer treatment completed; child should not be older than 18 years of age at the time of data collection; and parent should have sufficient knowledge of the Swedish language to complete the questionnaire. On the basis of these inclusion criteria, we invited the participation of both the mothers and fathers of all children who were diagnosed with malignant brain tumour 2000 - 2004 at the pediatric cancer unit at Astrid Lindgren Children's Hospital in Stockholm, Sweden.

A total of 69 parents were eligible and were invited to the first assessment (T1). Four of the parents declined to take part in the study when initially invited. Another six parents who agreed to participate did not return their questionnaires. Accordingly, the final study group contained 59 parents, leaving a response rate of $86 \%$ at $\mathrm{T} 1$. At T2, six of these 59 parents were not included due to relapse of the child's disease. Of the 53 eligible, 44 returned their T2-questionnaires. Thus, at T2 the respondents represented $83 \%$ of the parents eligible at $\mathrm{T} 2$, and $64 \%$ of the parents eligible at $\mathrm{T} 1$.

The study sample in the present paper included both mothers and fathers from 19 families, five mothers from families where the fathers had not responded, and one father from a family where the mother had not responded.

Fifty-five per cent of the parents $(n=24)$ in the study had children diagnosed with low-grade astrocytoma, $18 \%(n=8)$ medulloblastoma, and 11\% $(n=5)$ opticusglioma. The children of the remaining parents had been diagnosed with teratoma ( 2 parents), oligodendromatosis ( 2 parents), chraniopharyngeoma (2 parents), and dysgerminoma (1 parent). The time that had elapsed from the disclosure of the child's cancer diagnosis to study assessment ranged from 17 to 71 months (mean 42 months). Cancer treatment was completed 8 to 70 months prior to assessment (mean 35 months). The age of the child at data collection ranged from 5 to 18 years (mean 11 years). General demographic information about the parents in the study group is presented in Table 1.

Reference data were collected from parents in the general community. Inclusion criteria were: being a parent of at least one child between 0 and 16 years; residence in the municipality of Stockholm (like the respondents in the study sample). 450 randomly selected parents were invited, of whom $50 \%$ were fathers and $50 \%$ were mothers. Two parents could not be reached at the stated address. Of the remaining 448 parents 294 completed the questionnaire, and accordingly the response rate was $66 \%$. In the present study, the reference group was set to include only parents of children with no serious or chronic diseases. Therefore the following questionnaires were excluded from the entire reference group: 14 parents of children currently suffering from serious or chronic diseases, 13 parents of children who had previously suffered from a serious or chronic disease, and 5 parents who had not submitted data regarding their children's diseases. The remaining sample included 259 parents, of whom $107(41 \%)$ were fathers and 152 (59\%) were mothers. Demographic information about the participants in the reference group is also presented in Table 1. 
Table I. Demographic data for parents of children after completed treatment for CNS tumour, and reference parents.

\begin{tabular}{lll}
\hline & Study parents, \% & Reference parents, \% \\
\hline $\begin{array}{l}\text { Education } \\
\text { elementary school only }\end{array}$ & 2 & 4 \\
college/high school & 66 & 34 \\
university & 32 & 62 \\
& & \\
Swedish-born & 80 & 80 \\
immigrants to Sweden & 20 & 20 \\
& & \\
Occupation/employment & 57 & 57 \\
working full-time & 23 & 16 \\
working part-time & 5 & 12 \\
on parental leave & 2 & 3 \\
unemployed & 13 & 12 \\
other & & \\
Children's age; at least one child... & & 66 \\
up to and including 6 years & 27 & 36 \\
$7-12$ years & 75 & 30 \\
13-18 years & 61 & 11 \\
over 18 years & 9 & Reference parents, \\
& & mean (min-max) \\
& Study parents, & $40(22-64)$ \\
Parent's age, years & mean (min-max $)$ & $1.9(1-5)$ \\
Number of children in the family & $2.7(1-5)$ & \\
& & \\
& &
\end{tabular}

\section{Procedures}

Data were collected through self-report questionnaires that respondents completed at home. Parents in the study group were invited to participate by telephone by a contact nurse. Those who agreed to participate were sent a letter with written information about the study, the questionnaire, and a prepaid reply envelope. As was notified in the information letter, T2-questionnaires were sent out approximately 7 months after the completion of the T1-questionnaires. A series of reminders were sent for both T1 and T2. Parents who had not completed and returned their questionnaires two weeks after the distribution received a reminder. This procedure was repeated after another two weeks. For the reference group, a random sample of addresses was obtained from the national register of personal addresses (SPAR, Statliga personadressregistret). Reference parents received a postal invitation including a letter describing the study, the questionnaire, a pre-paid reply envelope, and a slip which parents could return if they chose not to participate in the study. Reminder letters were sent on three occasions to those who at that time had not returned either a completed questionnaire or a slip stating that they did not want to participate. The three occasions were one, three and six weeks after the initial distribution of invitations.

The study was approved by the Ethics Committee at Karolinska Institutet, Stockholm, Sweden. 


\section{Assessment of burnout}

The Shirom-Melamed Burnout Questionnaire (SMBQ; Grossi, Perski, Evengard, Blomkvist, \& OrthGomer, 2003; Melamed et al., 1992; Melamed et al., 1999) was used to assess burnout. This instrument contains 22 items that measure different facets of the burnout syndrome, as expressed by four subscales: emotional exhaustion and physical fatigue (8 items; below referred to as exhaustion/fatigue), listlessness (4 items), tension (4 items), and cognitive difficulties (6 items). The response format is a Likert scale graded 1 to 7 . Mean scores constitute the individual results, with a higher score reflecting more burnout symptoms. In the present study, scores for each subscale, as well as an overall burnout index (SMBQ-total), were calculated for each participant.

Satisfactory to good internal consistency of the instrument in the present study was indicated for the SMBQ-total as well as for the four subscales: The Cronbach's alpha values were for SMBQ-total in the study group 0.98 , and in the reference group 0.95 . For the subscales, the alpha values were: exhaustion/fatigue 0.93 (study group) and 0.90 (reference group); listlessness 0.89 and 0.84 ; tension 0.89 and 0.78 ; and cognitive difficulties 0.97 and 0.94 .

\section{Data management}

Respondents who failed to answer more than $25 \%$ of the items in a SMBQ subscale were excluded from analyses employing that subscale. For parents with $25 \%$ or fewer items unanswered, missing values were replaced with the individual mean score of the scale in question. In the study group, such imputations were made for one respondent's answer to an item in the exhaustion/fatigue subscale, and for one and two respondents' answers to items in the listlessness and tension subscales, respectively. The self-reported burnout was analyzed in two ways:

1) Quantity of burnout symptoms in the form of the individual SMBQ-total and subscale scores represented relative burnout;

2) A measure of current burnout was obtained through categorizing the SMBQ-total scores. This procedure was based on the cut-off levels set by Grossi et al. (2003): respondents with a score of 2.75 or less were assigned to a low-burnout group, while a score of 3.75 or more indicated high-burnout. In the present study these two categories are referred to as no burnout, including those reporting no or negligible expressions of burnout symptoms, and current burnout, which can be assumed to indicate evident symptoms of burnout. These two categories thus included only the respondents with the lowest and the highest SMBQ-scores.

\section{Statistical analyses}

Relative burnout in the study group was compared with that among reference parents: one-way analysis of variance (ANOVA) was used to compare the two groups regarding scores of SMBQ-total and the four subscales. Frequency of current burnout and no burnout is presented; comparisons of incidence in the study group and the reference group were made using a Chi-Square test.

Gender effects were addressed by means of paired-samples t-tests, which were used to compare levels of relative burnout in mother and father from the 19 couples where both parents had participated in the study. Thus, separate paired-samples analyses were made regarding SMBQ-total and each of the four subscales. Correlations of burnout scores in the mothers and fathers from the couples were also calculated, in order to check for any systematic covariation.

The variation in relative burnout in relation to time elapsed post-treatment was examined using Pearson correlation analyses (2-tailed). Correlation analyses were carried out separately for mothers and fathers.

\section{Results}

Mean and standard deviation (SD) values for SMBQ and its subscales are presented in Table 2. SMBQ-total scores ranged from 1.27 to 6.95 in the study group. Among reference parents, minimum and maximum scores for SMBQ-total were 1.00 and 6.55, respectively. Skewness values ranging from 0.29 to 0.65 indicated a normal distribution of scores in SMBQ-total and all subscales in both groups. 
Table II. SMBQ scores of parents of children after completed treatment for CNS tumour, and reference parents.

\begin{tabular}{|c|c|c|c|c|}
\hline & \multicolumn{2}{|c|}{ Study parents, Mean (SD) } & \multicolumn{2}{|c|}{ Reference parents, Mean (SD) } \\
\hline & $\begin{array}{l}\text { Mothers } \\
(n=24)\end{array}$ & Fathers $(n=20)$ & Mothers $(\mathrm{n}=152)$ & Fathers $(n=107)$ \\
\hline Burnout (SMBQ-total) & $3.94(1,50)$ & $3.01(1.47)$ & $3.26(1.24)$ & $2.80(1.00)$ \\
\hline Exhaustion/ fatigue & $4.02(1.38)$ & $2.95(1.60)$ & $3.22(1.40)$ & $2.78(1.16)$ \\
\hline Listlessness $^{\text {a }}$ & $4.05(1.40)$ & $3.61(1.53)$ & $3.46(1.35)$ & $3.20(1.15)$ \\
\hline Tension $^{\mathrm{b}}$ & $3.92(1.84)$ & $3.22(1.52)$ & $3.41(1.36)$ & $3.04(1.16)$ \\
\hline Cognitive difficulties & $3.77(1.89)$ & $2.58(1.56)$ & $3.03(1.54)$ & $2.45(1.26)$ \\
\hline
\end{tabular}

Exclusions of questionnaires due to missing data:

${ }^{a}$ one study mother, two reference fathers, and six reference mothers.

${ }^{\mathrm{b}}$ one study father, one study mother, three reference fathers, and one reference mother.

Relative burnout. Mothers in the study group reported consistently more relative burnout than reference mothers (Table 2; Figure 1). However, the differences were significant only for SMBQ-total $(F=5.60 ; p=.015)$ and the aspects of exhaustion/fatigue $(F=6.77 ; p=.010)$ and cognitive difficulties $(F=4.44 ; p=.037)$. The difference regarding listlessness was approaching significance $(F=3.78 ; p=.053)$.

For fathers, the ANOVAs indicated no differences between the study group and the reference group (Table 2; Figure 1).
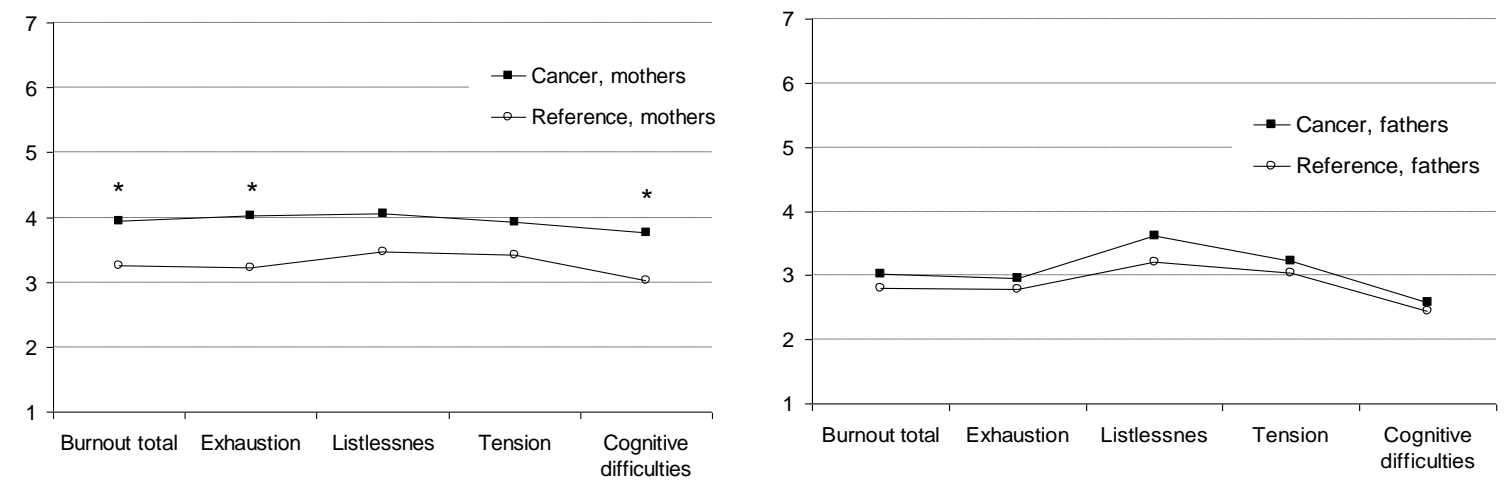

Fig. 1 Burnout in mothers and fathers of children treated for brain tumour and in reference mothers and fathers. $* \mathrm{p}<0.05$

Current burnout. Among the mothers in the study group, 54\% $(n=13)$ were assigned to the current burnout group, and $25 \%(n=6)$ reported no burnout. In the sample of reference mothers current burnout was less common (34\%), while no burnout was more frequent (38\%).

Twenty per cent $(n=4)$ of the fathers in the study group displayed current burnout, and $45 \%(n=9)$ of them reported no burnout. Among reference fathers, the corresponding incidences were 19\% current burnout and $52 \%$ no burnout. The differences between the groups were not statistically detectable. 
Parent gender. Paired-samples analyses of relative burnout in 19 couples in the study group revealed that mean scores of mothers were consistently higher than those of fathers regarding SMBQ-total $(t=$ $2.87, p=.010)$, exhaustion/fatigue $(t=3.15, p=.006)$, and cognitive difficulties $(t=3.05, p=0.007)$. No systematic gender difference within the parent couples was found for listlessness and tension. Correlation analysis of burnout scores in mothers and fathers from the couples indicated no systematic covariation.

Time post-treatment. The cross-sectional analyses revealed no statistically significant impact of the time that had elapsed since completion of treatment on relative burnout regarding mothers and fathers.

\section{Discussion}

The present paper reports the results from an evaluation of burnout among twenty-four mothers and twenty fathers of brain tumour survivors. More than half of the mothers reported current burnout, according to the criterion employed in the study. Symptoms of burnout occurred consistently more often among the mothers in the study group, compared with mothers of children with no history of serious or chronic diseases. The higher frequency of burnout symptoms among the study group mothers involved primarily emotional exhaustion and physical fatigue, and cognitive difficulties. Regarding fathers, no relation between burnout and being a parent of a brain tumour survivor was distinguished, despite a non-significant tendency to higher symptom levels in the study group. Parental stressors after completion of a child's treatment for brain tumour have not been comprehensively documented. Yet the everyday life of the family often involves several potentially stressful conditions and demands. In a study by Barr and colleagues (1999) comprising 44 children who had survived brain tumours, only one-fifth were completely free of morbidity. About one third are reported to suffer from moderate or severe neurological deficits (Anderson, 2003). Weiss (2002) notes that mental retardation and autism in a child may predict burnout in mothers (no fathers were included in the study). Regarding brain tumour survivors, it can be assumed that both serious dysfunctions and minor deficits such as cosmetic changes may influence the everyday life of the former patients (Schwartz, 1999), and consequently also of the parents.

In the present study, the mother was more often the parent experiencing the highest rate of burnout symptoms. At the same time, no significant correlation between mothers' and fathers' burnout levels (within couples) were found. This indicated that although the mother and father may have several shared concerns regarding the child and the family, these shared concerns were not the main or only source of burnout symptoms. The higher levels of burnout among women compared with men are in line with previous findings in other settings (Lindblom, Linton, Fedeli, \& Bryngelsson, 2006; Shirom et al., 1997). This difference may reflect a gender typical style in responding to the questionnaire. However, the fact that burnout in mothers but not in fathers differed from that in reference parents implies that mothers of brain tumour survivors indeed experience chronic stress to a greater extent than the fathers. A tentative explanation of this gender difference is that mothers have frequently taken on a heavier parenting burden than the fathers, as mothers traditionally tend to do (Milgram \& Atzil, 1988). It has also been argued that the parent role of mothers is often associated with the stress of excess expectations from others (Jackson \& Mannix, 2004). Furthermore, women are assumed to be particularly at risk for the stress of multiple roles (Orth-Gomer \& Leineweber, 2005), and chronic strain related to combining parent role and work is a predictor of distress in mothers (Naerde, Tambs, Mathiesen, Dalgard, \& Samuelsen, 2000).

Nonetheless, despite the need to monitor mothers' parenting burdens in particular, professionals should be encouraged to consider individual variation rather than to presuppose the existence of gender differences, as the latter may lead to false assumptions about psychosocial needs. Indeed, it was a father who reported the maximum burnout-total score in the present study group. Certainly fathers do react to a child's cancer (Chesler \& Parry, 2001), although burnout may be more common among mothers.

As noted in the stress literature, chronic stress is most often associated with excessive demand, although it can arise from other persistent conditions such as threat, uncertainty, or restriction of choice (Wheaton, 1997). Strain does not have to be traumatic or severe to have high psychological impact (Singer \& Davidson, 1986). Even low-intensity stressors may create a severe effect, if they are long-lasting or recurrent. Furthermore, each single stressor need not persist for long, as it has been 
suggested that separate stressors may give rise to cumulative effects. Each exposure to such a stressor may constitute a minimum of threat, but if the strain persists the impact may accumulate over time, and lead to exhaustion (Singer \& Davidson, 1986). The stresses of parenting a child with special needs probably augment any strain in other areas, for example at work.

Among the parents in this study, burnout symptoms were not related to time elapsed since the end of treatment. This suggests that possible disease-related stressors may have similar impact during the years after treatment completion. However, individual trajectories of stress over time have to be investigated longitudinally.

The findings of this study argue for the psychosocial follow-up of families after a child's brain tumour to address chronic stress and its effects. To prevent psychological exhaustion, chronic stressors should be identified and reduced or eliminated (Swedish National Board of Health and Welfare, 2003). Parents may need support in this process. As regards full-blown burnout, patients appear to benefit from a combination of medical, paramedical, and psychological interventions, modified to suit the needs of the individual (Perski \& Grossi, 2004).

\section{Limitations}

Some methodological limitations of this study need to be recognized. First, the present study did not investigate the source of any chronic stress, and, consequently, assumptions about the causes of burnout can only be tentative. A longitudinal approach following individual cases would be necessary to verify or refute hypotheses about circumstances leading to burnout in the respondents. In addition, the number of parents in the study was relatively small, limiting the possibilities of drawing conclusions.

Furthermore, it should be noted that a low relative burnout, i.e. a low SMBQ-score, means "far from being burned-out" rather than "slightly burned-out". Moreover, in the absence of generally accepted diagnostic criteria, it can be questioned whether "current burnout" as it was defined in this study really is serious enough to be regarded as clinically significant. However, comparison with a clinical sample supports this assumption: The mean SMBQ score among the study parents with "current burnout" in this study was 5.14 (SD 1.0), which corresponds to the mean SMBQ score of 5.37 in a sample of men and women on sick leave due to burnout (Grossi et al., 2005).

Finally, in this study one single measure of burnout was used. The use of additional assessment modalities, e.g. physiological correlates, would provide a broader and, perhaps, more reliable basis for conclusions.

\section{Conclusions \& Future}

To summarize, the outcome of the present study implies that the burnout found among the parents in the study group may, to a certain extent, be associated with parenting a brain tumour survivor, at least in regards to the mothers. The situation of the family appears not to affect fathers in the same way. The findings encourage further studies of chronic stress and psychological exhaustion among parents after a child's cancer treatment. A thorough examination of parents' burdens during the period of convalescence and rehabilitation after childhood brain tumour should facilitate identification of factors associated with the incidence of burnout. The situation of parents of children with other serious or chronic diseases should also be considered. Furthermore, the findings highlight the necessity of paying attention to signs of chronic stress in the psychosocial follow up of these families.

\section{Acknowledgements}

I gratefully acknowledge the time and energy contributed by the participating parents. Thanks also to Anncarin Pal, RN, who assisted in the process of inviting parents to participate in the study group. The study was supported by the Children's Cancer Foundation of Sweden (RKT05/006). 


\section{References}

Anderson, N. E. (2003). Late complications in childhood central nervous system tumour survivors. Current Opinion in Neurology, 16, 677-683.

Barakat, L. P., Kazak, A. E., Meadows, A. T., Casey, R., Meeske, K., \& Stuber, M. L. (1997). Families surviving childhood cancer: A comparison of posttraumatic stress symptoms with families of healthy children. Journal of Pediatric Psychology, 22, 843-859.

Barr, R. D., Simpson, T., Whitton, A., Rush, B., Furlong, W., \& Feeny, D. H. (1999). Health-related quality of life in survivors of tumours of the central nervous system in childhood--a preference-based approach to measurement in a cross-sectional study. European Journal of Cancer, 35, 248-255. Barrera, M., D'Agostino, N. M., Gibson, J., Gilbert, T., Weksberg, R., \& Malkin, D. (2004). Predictors and mediators of psychological adjustment in mothers of children newly diagnosed with cancer. Psycho-Oncology, 13, 630-641.

Brown, R. T., Madan-Swain, A., \& Lambert, R. (2003). Posttraumatic stress symptoms in adolescent survivors of childhood cancer and their mothers. Journal of Traumatic Stress, 16, 309-318.

Carpentieri, S. C., Meyer, E. A., Delaney, B. L., Victoria, M. L., Gannon, B. K., Doyle, J. M., et al. (2003). Psychosocial and behavioral functioning among pediatric brain tumor survivors. Journal of Neuro-Oncology, 63, 279-287.

Carpentieri, S. C., Waber, D. P., Pomeroy, S. L., Scott, R. M., Goumnerova, L. C., Kieran, M. W., et al. (2003). Neuropsychological functioning after surgery in children treated for brain tumor.

Neurosurgery, 52, 1348-1356; discussion 1356-1347.

Chesler, M. A., \& Parry, C. (2001). Gender roles and/or styles in crisis: an integrative analysis of the experiences of fathers of children with cancer. Qualitative Health Research, 11, 363-384.

Duffner, P. K. (2004). Long-term effects of radiation therapy on cognitive and endocrine function in children with leukemia and brain tumors. Neurologist, 10, 293-310.

Forinder, U. (2004). Bone marrow transplantation from a parental perspective. Journal of Child Health Care, 8, 134-148.

Frank, N. C., Brown, R. T., Blount, R. L., \& Bunke, V. (2001). Predictors of affective responses of mothers and fathers of children with cancer. Psycho-Oncology, 10, 293-304.

Fuemmeler, B. F., Mullins, L. L., \& Marx, B. P. (2001). Posttraumatic stress and general distress among parents of children surviving a brain tumor. Child Health Care, 30.

Grootenhuis, M. A., \& Last, B. F. (1997). Predictors of parental emotional adjustment to childhood cancer. Psycho-Oncology, 6, 115-128.

Grossi, G., Perski, A., Ekstedt, M., Johansson, T., Lindstrom, M., \& Holm, K. (2005). The morning salivary cortisol response in burnout. Journal of Psychosomatic Research, 59, 103-111.

Grossi, G., Perski, A., Evengard, B., Blomkvist, V., \& Orth-Gomer, K. (2003). Physiological correlates of burnout among women. Journal of Psychosomatic Research, 55, 309-316.

Hoekstra-Weebers, J. E., Jaspers, J. P., Kamps, W. A., \& Klip, E. C. (2001). Psychological adaptation and social support of parents of pediatric cancer patients: a prospective longitudinal study. Journal of Pediatric Psychology, 26, 225-235.

Jackson, D., \& Mannix, J. (2004). Giving voice to the burden of blame: a feminist study of mothers' experiences of mother blaming. International Journal of Nursing Practice, 10, 150-158.

Kazak, A. E., Alderfer, M., Rourke, M. T., Simms, S., Streisand, R., \& Grossman, J. R. (2004). Posttraumatic stress disorder (PTSD) and posttraumatic stress symptoms (PTSS) in families of adolescent childhood cancer survivors. Journal of Pediatric Psychology, 29, 211-219.

Kazak, A. E., Barakat L P, Meeske K, Christakis D, Meadows A T, Casey R, et al. (1997).

Posttraumatic stress, family functioning, and social support in survivors of childhood leukemia and their mothers and fathers. Journal of Consulting and Clinical Psychology, 65, 120-129.

Koocher, G. P., \& O'Malley, J. E. (1981). The Damocles syndrome: psychosocial consequences of surviving childhood cancer. New York, NY: McGraw-Hill.

Leventhal-Belfer, L., Bakker, A. M., \& Russo, C. L. (1993). Parents of childhood cancer survivors: A descriptive look at their concerns and needs. Journal of Psychosocial Oncology, 11, 19-41.

Lindahl Norberg, A., Lindblad, F., \& Boman, K. K. (2005). Parental traumatic stress during and after paediatric cancer treatment. Acta Oncologica, 44, 382-388. 
Lindblom, K. M., Linton, S. J., Fedeli, C., \& Bryngelsson, I. L. (2006). Burnout in the working population: relations to psychosocial work factors. International Journal of Behavioral Medicine, 13, 51-59.

Mabbott, D. J., Spiegler, B. J., Greenberg, M. L., Rutka, J. T., Hyder, D. J., \& Bouffet, E. (2005). Serial evaluation of academic and behavioral outcome after treatment with cranial radiation in childhood. Journal of Clinical Oncology, 23, 2256-2263.

Maslach, C., Schaufeli, W. B., \& Leiter, M. P. (2001). Job burnout. Annual Review of Psychology, 52, 397-422.

Melamed, S., Kushnir, T., \& Shirom, A. (1992). Burnout and risk factors for cardiovascular diseases. Behavioral Medicine, 18, 53-60.

Melamed, S., Ugarten, U., Shirom, A., Kahana, L., Lerman, Y., \& Froom, P. (1999). Chronic burnout, somatic arousal and elevated salivary cortisol levels. Journal of Psychosomatic Research, 46, 591598.

Milgram, N. A., \& Atzil, M. (1988). Parenting stress in raising autistic children. Journal of Autism and Developmental Disorders, 18, 415-424.

Miller, G. E., Cohen, S., \& Ritchey, A. K. (2002). Chronic psychological stress and the regulation of pro-inflammatory cytokines: A glucocorticoid-resistance model. Health Psychology, 21, 531-541. Mulhern, R. K., \& Butler, R. W. (2004). Neurocognitive sequelae of childhood cancers and their treatment. Pediatric Rehabilitation, 7, 1-14; discussion 15-16.

Mulhern, R. K., Merchant, T. E., Gajjar, A., Reddick, W. E., \& Kun, L. E. (2004). Late neurocognitive sequelae in survivors of brain tumours in childhood. Lancet Oncology, 5, 399-408. Mulhern, R. K., Palmer, S. L., Merchant, T. E., Wallace, D., Kocak, M., Brouwers, P., et al. (2005). Neurocognitive consequences of risk-adapted therapy for childhood medulloblastoma. Journal of Clinical Oncology, 23, 5511-5519.

Naerde, A., Tambs, K., Mathiesen, K. S., Dalgard, O. S., \& Samuelsen, S. O. (2000). Symptoms of anxiety and depression among mothers of pre-school children: effect of chronic strain related to children and child care-taking. Journal of Affective Disorders, 58, 181-199.

Orth-Gomer, K., \& Leineweber, C. (2005). Multiple stressors and coronary disease in women. The Stockholm Female Coronary Risk Study. Biological Psychology, 69, 57-66.

Perski, A., \& Grossi, G. (2004). [Treatment of patients on long-term sick leave because of stressrelated problems. Results from an intervention study] Swedish. Lakartidningen, 101, 1295-1298. Phipps, S., Long, A., Hudson, M., \& Rai, S. N. (2005). Symptoms of post-traumatic stress in children with cancer and their parents: Effects of informant and time from diagnosis. Pediatric Blood Cancer, 45, 952-959.

Quin, S. (2004). The long-term psychosocial effects of cancer diagnosis and treatment on children and their families. Social Work Health Care, 39, 129-149.

Radcliffe, J., Bennett, D., Kazak, A. E., Foley, B., \& Phillips, P. C. (1996). Adjustment in childhood brain tumor survival: child, mother, and teacher report. Journal of Pediatric Psychology, 21, 529-539. Sawyer, M., Antoniou, G., Toogood, I., Rice, M., \& Baghurst, P. (2000). Childhood cancer: a 4-year prospective study of the psychological adjustment of children and parents. Journal of Pediatric Hematology/Oncology, 22, 214-220.

Schwartz, C. L. (1999). Long-term survivors of childhood cancer: the late effects of therapy. The Oncologist, 4, 45-54.

Shirom, A., Westman, M., Shamai, O., \& Carel, R. S. (1997). Effects of work overload and burnout on cholesterol and triglycerides levels: the moderating effects of emotional reactivity among male and female employees. Journal of Occupational Health Psychology, 2, 275-288.

Singer, J. E., \& Davidson, L. M. (1986). Specificity and Stress Research. In M. H. Appley \& R. Trumbull (Eds.), Dynamics of stress: Physiological, psychological, and social perspectives (pp. 4761). New York: Plenum Press.

Strike, P. C., \& Steptoe, A. (2004). Psychosocial factors in the development of coronary artery disease. Progress in Cardiovascular Ddiseases, 46, 337-347.

Swedish National Board of Health and Welfare. (2003). [Syndrome of exhaustion. Stress related psychological ill-health] Swedish (No. 2003-123-18). Stockholm, Sweden. 
Toker, S., Shirom, A., Shapira, I., Berliner, S., \& Melamed, S. (2005). The association between burnout, depression, anxiety, and inflammation biomarkers: C-reactive protein and fibrinogen in men and women. Journal of Occupational Health Psychology, 10, 344-362.

Van Dongen-Melman, J. E. W. M., Pruyn, J. F. A., De Groot, A., Koot, H. M., Hahlen, K., \& Verhulst, F. C. (1995). Late psychosocial consequences for parents of children who survived cancer. Journal of Pediatric Psychology, 20, 567-586.

Van Dongen-Melman, J. E. W. M., Van Zuuren, F. J., \& Verhulst, F. C. (1998). Experiences of parents of childhood cancer survivors: a qualitative analysis. Patient Education and Counseling, 34, 185-200.

van't Hooft, I. V., Andersson, K., Bergman, B., Sejersen, T., Von Wendt, L., \& Bartfai, A. (2005). Beneficial effect from a cognitive training programme on children with acquired brain injuries demonstrated in a controlled study. Brain Injury, 19, 511-518.

Weiss, M. J. (2002). Hardiness and social support as predictors of stress in mothers of typical children, children with autism, and children with mental retardation. Autism, 6, 115-130.

Wheaton, B. (1997). The nature of chronic stress. In B. H. Gottlieb (Ed.), Coping with chronic stress (pp. 43-73). New York: Plenum Press.

Wijnberg-Williams, B. J., Kamps, W. A., Klip, E. C., \& Hoekstra-Weebers, J. E. (2006).

Psychological adjustment of parents of pediatric cancer patients revisited: five years later. PsychoOncology, 15, 1-8. 